18. Perfectly cured; she can, with the aid of the adjoining fingers, flex and extend the finger at pleasure; she can also use it at the piano, and grasp objects firmly.

Aberdeen, Dec. 3, 1841.

\section{CONTRIBUTIONS TO MEDICINE.}

\author{
By James B. Thompson, A.B., M.D., \\ Surgeon, \&c. \\ "chorea sancti viti,". With cases and \\ OBSERVATIONS.
}

Utility of the Secale Cornutum, or Ergot of Rye, in Chorea; Paralysis from Lead, and the Paralysis Agituns or Tremens in Elderly People; Treatment, \&c. \&c.

Frrst Case.-Having some time since been called to see an interesting little girl, atat. 6 , who had suffered from this disease for six weeks previously; she was of a scrofulous diathesis, and evidently affected with worms; $I$ tried mild emetic and aperient medicines at first, and afterwards put her on the mercury with chalk powders with the senna and scammony powder's alternately, allowed her a very nutritious diet, not exciting nor stimulating. She had the disease in a very marked form, principally confined to the left arm and leg, and was generally seized with it in the afternoon; the circulation was not much affected unless during the paroxysm of the attack, which seldom lasted longer than twelve or fifteen minutes. Faving attributed the disease in this case to the presence of worms, with the constitutional affection above alluded to, $I$ attended principally to the improving the general health and removing the exciting cause, which afterwards proved to be the worms, for when oil of turpentine was given, large quantities of slimy, muco-purulent-like collections of darkishcoloured stuff were passed, and in this was observed at each stool, often in considerable number, the kind of worms usually present in the intestinal canal. This treatment was continued for about a fortnight, when a more tonic plan was pursued, occasionally returning to the powders and turpentine. The child eventually got well, and has not had any return of the disease for the last twelve months, and is considerably improved in her general health.

SeCond Case-The next case is one of more interest, as it occurred at a more advanced period of life, and in which a more complicated treatment was requisite. It occurred in a young lady, ætat. 16, of leucophlegmatic temperament; she was labouring under it for eighteen months previously to my seeing her, and was under the care of the family medical man, who had tried the usual plans suggested, namely, emetics, purga- tives, emmenagogues, \&c. In this case the right arm and lower extremity were affected, and occasionally the muscles of the neck and of deglutition became engaged during the attack, which was more frequent, often twice in the twenty-four hours, and lasted sometimes nearly from twenty minutes to half an hour, during which time the arm would be tossed across the chest, and the leg involuntarily shook about in a manner most Iudicrous, but painful to witness. I directed in this case a course of bitters and tonics at first, occasionally giving an aloetic and myrrh pill after the prima via had been well and freely cleared out; ordered the hipbath and warm salt-water stupes and some leeches to the sacrum : we tried the carbonate of iron in powders. This line of treatment was pursued for nearly three weeks: there evidently was an improvement, insomuch that the attacks were become less frequent and milder, still they continued, and there was no apparent tendency to the catamenia; to the absence of which we attribute, in a great measure, the disease in this young lady's case. It was at this period that I was induced to recommend the "secale cornutum," and in the following manuer, which $l$ consider preferable to the usual mode of giving it in powders : I directed a drachm of the recentlypowdered ergot to be infused in three ounces of water, at boiling temperature, and allowed to infuse for twenty-five minutes; then adding to this a little new milk, which, I think, makes it more palatable, and less likely to be rejected or disagree with the stomach, which I have observed it do in many cases when given by way of powder. It was directed to be taken as follows; viz., a dessertspoonful three or four times a-day, gradually increasing it for a few days, and then returning to the previous tonic medicines. I gave in this case the sulphate of quinine in Madeira wine, which seems a real and agreeable way of giving it. This practice was continued for about a fortnight, when we were informed that there was a very slight appearance of the catamenia; but whether to attribute this to the ergot of rye, or to the previous treatment or both, I am not disposed to say. However, this young lady got well, and has not had the least appearance of this affection for the last two years, and is now a very healthy-looking subject.

\section{General Observations.}

The disease to which the name of "chorea sancti viti" was first applied, very nearly resembles that produced by the bite of the "tarantula." "rlhis disease in its simple form occurs more frequently in persons whose vital powers are depressed, the entire class of vital organs performing their functions imperfectly, and thereby occasioning an increased and morbid sensibility of the nervous system. This disease seems not only 
to disturb the functions of the nerves of of arsenic, jodine, hydriodate and ioduretted voluntary power, but also those parts from hydriodate of potash, oxide and sulphate of which these nerves originate in the cere- zinc, nitrate of silver, valerian, stramonium, bro-spinal axis. Chorea is much more fre- subnitrate of bismuth, turpentine, cajeput quent in the female than in the male subject; and tusk liver oil, dippels, animal oil, sulthe average ratio of its frequency is stated phur as a purgative, strychnine or nux voto be about three in the former to one in the latter sex. It presents itself as early as five and six years of age; but the most common period of life at which it occurs varies from seven to fifteen years, from second dentition to puberty; but no age is exempt from it. It has been witnessed in all ages, from forty to fifty, and even up to seventy and eighty. In advanced life it is mostly complicated with other affections, such as hemiplegia, rheumatism, paralysis, mental affections, \&c. \&c.

The predisposing causes are very numerous: hereditary predisposition, nervous temperament, constitutional debility from whatever cause, deficient nourishment, malformation, precocious development of the mental powers or functions, venery, weak assimilative or digestive powers, constipa tion, deranged secretions, inactive liver, vicissitudes of temperature, cold and moist climates, sedentary and confined occupations, bad air, ill-ventilated or crowded and close places, mephitic and miasmatic effluvia, want of cleanliness, rheumatic, scrofulous, and rickety diathesis. The exciting causes are worms, morbid or fæcal accumulations in the bowels, falls or injuries to the spine or brain, fright, incautious use of mercurial or lead preparations, suppressed discharges and eruptions, as herpes, itch, \&c., metastasis and extension of rheumatism to the membranes of the spinal cord, previous disease, particularly of an eruptive nature, epilepsy, hysteria, second dentition, anxiety, concealed mental emotions or impressions, jealousy, envy, amenorrhoea, dysmenorrhoea.

Treatment.-In no one disease in the whole range of the practice of medicine has there been such a great variety of remedies recommended as in the disease now under consideration; but a close and careful consideration of the nature of the case will readily suggest a sound and rational line of practice. This ought and should be the ruling principle adhered to in the treatment of all diseases. The remedies generally may be classed as follows; viz., emetics, 'aperients, purgatives, bitters, tonics, counter-irritation, by blisters, setons, issues, moxas, pustulation, electricity, galvanism, anthelmintics, ${ }^{*}$ emmenagogues, antispasmodics, narcotics, sedatives, mineral acids, prussic acid, Fowler's solution

* Anthelmintic. - Amonost the poorer classes in Ireland and in Scotland it is a common practice to get the husk of oats and burn it, and reduce it to a fine powder, then give it to the children in the morning with powdered sugar or in molasses. It seems to act as a mechanical agent, and may be use-

No. 961. mica, cold affusion, douche, warm and saltwater baths, ice along the spinal column, snake root, aromatic embrocations and liniments; blisters and general blood-letting have been found rather prejudicial than otherwise; local bleeding by means of leeches has proved useful.

The object in all these cases should be to get rid of morbid secretions and fæcal accumulations, the invariable cause of irritation to the organic nerves; to subdue and remove any vascular excitement or congestion of the vessels of the brain or spinal marrow, should symptoms indicate their presence. Toimprove the general health, and thereby raise the tone and energy of the general nervous system, and the natural healthy functions of the assimilating and secreting organs, irregular forms of chorea present more or less of a hysteric character, and these cases are sure to be attended with deranged and disordered states of the functions of the uterus, and of the circulation in the brain and spinal cord, or both. During convalescence, and in the advanced course of treatment, change of air, agreeable society, and amusement; exercise in the open air, on horseback, or in a carriage; the use of chalybeate or aperient mineral waters, the Cheltenham salts, with particular attention to the bowels and digestive organs generally.

It may be necessary to state why I was desirous of trying the ergot of rye in this disease. I did so on two grounds; first, reasoning from its well-known and long-established property of acting on the uterine system, and as the case recorded was one where $I$ deemed it advisable on this principle, I gave it with a view to predispose to the appearance of the catamenia; secondly, from its recently-discovered property of acting beneficially in cases of paralysis from lead, and in the paralysis agitans or tremens in advanced life, where $I$ have seen it of considerable benefit recently myself. It appears very difficult to account for the modus operandi of this medicine, for its effects are produced so rapidly in some cases, particularly of inaction of the uterus, that we cannot suppose it could have time to act thus through the medium of the circulation. It would occur to a person that if it were capable of acting at once on the sentient fibrillæ of the nerves of voluntary power, and thus on the muscles,

ful after a brisk purgative, which assists in the removal of the tenacious, slimy, mucus accumulations which are always present, and which afford a nidus for the worms to lie in. Rue-tea is also a very common remedy with these people. 
as in the cases of paralysis from lead; but more so in the "paralysis tremens," where it would seem to convey a more fixed steadiness and firmness; in these latter instances, this medicine can be given to a larger amount than is generally supposed; but it is one of those medicines that are apt to accumulate in the system, and hence a necessity for caution when using it. It was my being aware of this property that induced me to discontinue its use for a few days occasionally, and then give the tonic medicines alternately. This is I consider a desirable way of trying its utility in similar cases, and obviates in my mind any dangerous results from its accumulating in the patient's system. Though one case cannot be deemed fully satisfactory as to the utility of this or any other medicine, still I apprehend we know sufficient of its medicinal virtues to consider it as deserving of further notice and fair trial from the profession, in many of those cases, more particularly amongst females presenting themselves at public institutions suffering from a variety of complicated complaints, hysteria, chlorosis, epilepsy, \&c. It has been latterly given to a very considerable extent at St. Thomas's Hospital in cases of paralysis from lead; often as much as ten grains three times a-day for a month; and Dr. Williams asserts without the least inconvenience or bad effects, and with comparative success, where other remedies have failed. To try it fairly, then, we should have it recently powdered, and ascertain that it possesses its peculiar smell (resembling somewhat that of new-mown hay), and that it is not musty. Even with these precautions it occasionally may not succeed, certain constitutions appearing not to be susceptible of its influence. In some instances where it may be given to a large amount, it is likely to produce delirium, and it invariably seems to depress the pulse. I some years ago saw it in one instance, where it was given for inertness of the uterus, cause coma and stertorous breathing; but this was where it was given incautiously, and to a great extent; but no fatal consequences followed. It is certainly inadmissible in cases where there may be a predisposition to plethora or fulness about the head, neck, or chest.

In the "Journal de Chimie Medicale," M. Bonjean relates the discovery of two different active principles; one which acts as a poison is the oil of ergot, of an uniform consistence, an acrid flavour, a yellowish colour, soluble in cold ether and in boiling alcohol, it possesses poisonous properties in a high degree; the second active principle is the aqueous extract obtained by treating with water the powder, either deprived of its oil or not; it is brown, of a thick consistence, and musty-like smell; it is soluble in water, and can be formed into mixtures, syrups, pills, \&c.; it is not poisonous, but possesses very decided anti-hæmorrhagic properties. M. Blanc, of Aix les Baines, bears testimony to this latter anti-hæmorrhagic property.

33, Upper Gower-street, Jan. 6, 1842.

\section{EFFUSION OF BLOOD,}

UNDER THE

\section{MUCOUS MEMBRANE OF THE UVULA,} REMOVED BY INCISION.

Dr. Pauli, of Laudan, publishes three cases of this occurrence.

A man, thirty years old, enjoying good health, was taken whilst eating fish with a slight pain in the back of the throat, which he ascribed to the pricking of a fish-bone. A few minutes afterwards he suddenly lost his voice; a slight dyspnoea and difficulty of deglutition came on. The medical man observed that there existed on the uvula a small tumour of the size of a nut, of a bluish-red, which he recognised immediately as an extravasation of blood under the mucous membrane. It is probable that in this case the fish-bone had wounded a vessel of the velum, and that the blood gravitated into the uvula. M. Pauli opened this tumour, a little blood escaped, and the patient recovered his roice, and all the symptoms went away.

The second case was that of a healthy man, thirty-five years old. The patient attributed the symptoms which had occurred to the passage into the pharynx of the bone of a frog which he had been eating. The tumour was larger and the dyspnoea more severe than in the previous case. The incision produced a flow of blood, but not the immediate disappearance of all the inconveniences.

The woman who was the subject of the third case, was suddenly seized with aphonia after swallowing a hard crust of bread. She believed, like the two other patients, that this effect was due to apoplexy. This aphonia was preceded by an acute pain in the pharynx. M. Pauli recognised the tumour of the velum pendulum. A little incision removed all annoyance.

In general the incision of these little sanguineous tumours of the uvula suffices. A small incision endangers relapse when the vessel wounded continues to bleed, so that it becomes necessary to cut again. Furthermore, the incision and excision are scarcely painful, and the mucous membrane is quickly restored.

These cases had some circumstances in common. 1. They all believed themselves attacked with apoplexy. 2. They all lost the voice, and the respiration was difficult. They were calm and wrote intelligently all that occurred to them. 3. All these effects were produced by a mechanical cause. 4 . The three patients were easily relieved. Their cure would probably have occurred spon. 